\title{
Magnetization Heat in Superconductors and in Eddy Current problems: a Classical Thermodynamic Approach
}

\author{
Massimo Sorbi and Vittorio Marinozzi
}

\begin{abstract}
In the thermodynamic studies the problem of work done by magnetized materials is often approached with general-theorical approach. Sometimes ambiguities can be found in the demonstrations, because some authors do not specify clearly the terms in the relations. Conversely, a definition of "heat of magnetization" is not given in a general complete way. Some engineers and physicists use practical relations to calculate power dissipated on superconducting materials during magnetization cycles, but it is not possible to find theoretical works that justify the validity of these relations in general situations, for example as instantaneous losses. In this paper we want to approach both these aspects with a more complete analysis.
\end{abstract}

Index Terms - Heat exchange for magnetizing process, losses in superconductors, losses for eddy currents.

\section{INTRODUCTION}

$\mathrm{M}$ ANY thermodynamic texts and handbooks which treat the first principle of thermodynamics specify that among the works done on the system, there may be also the magnetic work. The volumetric magnetic work $l_{\mathrm{M}}$ is defined as:

$$
l_{M}=-\mu_{0} \int_{M_{1}}^{M_{2}} \vec{H} \cdot d \vec{M}
$$

where $H$ is the magnetic field and $M$ the magnetization of the body. Actually eq. (1) presents some ambiguities, because in presence of a magnetized body, the magnetic field $H$ may be the sum of the external field, usually called the "applied field" $H_{a}$, and the de-magnetizing field $H_{m}$ produced by the magnetized body itself: so an exact definition of $H$ is necessary. Some authors neglect to specify the origin of $H$ (for example treating only special configurations without de-magnetizing field [1-2]), whereas others explicitly distinguish the cases, arriving to conclude that $H$ is the "applied field" $H_{a}$ only [3-4].

If the work for magnetization is often discussed (even with some ambiguities sometimes, as focused above), the heat exchanged in magnetization processes is not afforded with a general approach. When some processes of energy dissipation are involved during closed cycles of magnetization, as for magnetic hysteresis in superconductors, most authors use practical relations. For example assuming to work in closed loops, for the energy conservation the volumetric heat absorbed $q_{M}$ is taken equal to the volumetric work received [5-6]; moreover, because the work is done in a closed cycle, in the usual case where

M. Sorbi and V. Marinozzi are with Milan University - Physics Dept. and INFN Sez. di Milano - LASA Lab., via fratelli Cervi 201, 20090 Segrate (Milan), Italy.
$\boldsymbol{M}$ and $\boldsymbol{H}$ are parallel, the integration variable can be substituted with the integrand, obtaining:

$$
q_{M}=-\mu_{0} \oint H d M=\mu_{0} \oint M d H
$$

The change in sign of last term in (2) is due to the fact that the path of integration changes verse when $H$ is exchanged with $M$.

Other authors demonstrate (2) with different approaches [7-8], and specify clearly that the magnetic field $H$ to be considered is the external applied field $H_{a}$. If we write $B_{a}=\mu_{0} H_{a}$ and because of a thermodynamic convention $q_{M}$ is the "absorbed heat", the "out-going heat" $q_{\text {diss }}$ (dissipated heat) can be re-written as:

$$
q_{\text {diss }}=-\oint M d B_{a}
$$

Many authors use (3) to calculate hysteresis losses in bulk and composite superconductors [5, 9-12].

However the eq. (2) and (3) result valid for a closed loop only, and they do not give information where and how much, along the cycle, the heat is exchanged. In other words, the average power per cycle can be computed, dividing the heat loss with the time period, but it is not possible to have information about the instantaneous losses.

Some other authors use to calculate superconducting hysteresis losses considering the dissipative work done by the pinning forces of fluxoid quanta during their displacement [13] (and again with this approach the average loss per cycle is calculated) or considering the volumetric power $\boldsymbol{J} \cdot \boldsymbol{E}[6,14]$. For this last method, it is necessary to assume that $\boldsymbol{J} \cdot \boldsymbol{E}$ is a dissipative power, as occurs for normal material and for the mixed state of IItype superconductors, and not a reversible power, as for the Meissner state [6]. An interesting review of classical methods used to calculate losses in HTS (mainly also valid in LTS) can be found in [8].

Here we want to give the demonstration of a different general method of calculating the instantaneous heat produced or absorbed during a magnetization process in superconductors or other problems of eddy currents, by means of a pure thermodynamic approach, that, to our knowledge, hasn't been presented in a general complete way.

\section{MAGNETIZATION WORK}

Firstly we want to re-propose a demonstration of the work done by a magnetic system against the external environment when it modifies its magnetization of a quantity $d \boldsymbol{M}$. With this demonstration we will reach the well-known relations of the magnetization work.

We initially suppose that the magnetic system, well defined from the rest of the universe, has a small volume 
$d V$, so that the magnetization $\boldsymbol{M}$ of the very small volume can be assumed uniform.

Clearly there is evidence that the elementary magnetic dipoles inside the system feel the influence of the magnetic field; without an external magnetic field, the work done by the system for the magnetization variation $d \boldsymbol{M}$ must be null. One may consider that the elementary dipoles themselves generate a magnetic field, and so a work may be done during the variation $d \boldsymbol{M}$ because of the interactions of the elementary dipoles with this "self-field". This is the situation that occurs when a magnetized volume generates a magnetic field $\boldsymbol{H}_{\boldsymbol{m}}$, usually called de-magnetizing field, due to the fictitious magnetizing currents inside the volume or on the surface, given by:

$$
\vec{J}_{M}=\operatorname{rot} \vec{M} \quad \vec{J}_{S M}=\vec{M} \times \vec{n}
$$

where $\boldsymbol{n}$ is the unitary vector normal to the volume surface, or, from a different but equivalent approach, due to the fictitious volumetric or superficial magnetic charges, given by:

$$
\rho_{M}=-\operatorname{div} \vec{M} \quad \sigma_{M}=\vec{M} \cdot \vec{n}
$$

Actually, the hypothetical work done by the elementary dipoles for the interaction with this "self-field" $\boldsymbol{H}_{\boldsymbol{m}}$ must not be considered here, because it is not a work done against external parts of the system, but eventually against internal parts of the system. According to formal definition of the works in thermodynamics, this kind of energy will have to be considered in the energetic balance of the internal energy, as we will discuss later.

In conclusion we may state that if the system does a work for the variation $d \boldsymbol{M}$, it is necessary the presence of an external magnetic field $\boldsymbol{H}_{\boldsymbol{a}}$ (also called the "applied field"), which is produced on the space occupied by the volume of the system independently by the presence of the system, or as the system was substituted by vacuum. Once we have chosen a small volume $d V$ for the system, we may consider again $\boldsymbol{H}_{\boldsymbol{a}}$ being uniform on $d V$.

We suppose now that the external applied magnetic field $\boldsymbol{H}_{\boldsymbol{a}}$ is generated by an ideal, very long, thin solenoid, in which the system is located. The solenoid has to be connected to a power supply, which keeps constant the current $I_{a}$ which circulates in the solenoid, in order to keep constant $\boldsymbol{H}_{\boldsymbol{a}}$ during the whole process which produce the magnetization variation $d \boldsymbol{M} .{ }^{1}$ The work $d L_{M}$ done by the

\footnotetext{
${ }^{1}$ The magnetic work done by the system has to be computed supposing that $H_{a}$ is constant during the process because $H_{a}$ represents a quantity produced by an external apparatus respect to the exanimated system; so the computation of the work has to be done by supposing that this external quantity does not vary. The situation is very similar to the computation of the mechanical work given by $d L=P d V$, where $P$ is the pressure produced by an external force, which is considered constant during the infinitesimal variation $d V$ of the volume. In the case of the work due to magnetization this concept apparently appears more hardly to be figured because the magnetization variation, in the real cases, is almost always produced by the variation of magnetic field external to the system. However, in order to apply correctly the thermodynamic concepts, it is necessary to decouple the works done by the system for the variation of its own magnetization only, from the other energies given by the external apparatus to vary the magnetic field $H_{a}$. The last ones, in fact, don't have to be considered as works done from the examined variation $d M$, because ideally they are performed externally and they are independent on the considered system. The magnetization variation may be represented as produced by relaxations of some internal forces between elementary dipoles, exactly as we are used to represent the volume variation of a gas in a container as due to particle collisions to the walls, and the particles lose kinetic internal energy when the recipient adiabatically expands.
}

magnetic system against the external, conceptually has to be found as the work $d L_{g}$ done by the power supply, i.e.:

$$
d L_{M}=-d L_{g}=-\Delta V \cdot I_{a} \cdot d t
$$

where $d t$ is the infinitely small time during the variation $d \boldsymbol{M}$ of the system and $\Delta V$ is the voltage across the terminal of the power supply, which is equal to the voltage across the solenoid. If we don't consider the power dissipated in the solenoid for Joule effect, which does not depend on the considerations about the magnetization that we are discussing here, the potential difference $\Delta V$ is zero if the magnetization variation is zero, because nothing is varying. Conversely, when there is a variation $d \boldsymbol{M}$ of the magnetization, an electro-motive force $\Delta V$ will be present in the terminals of the solenoid, which by definition can be computed as:

$$
\Delta V=-\int_{L} \vec{E} \cdot d \vec{l}
$$

with $L$ the path along the windings that compound the solenoid, and $\boldsymbol{E}$ the electric field (strictly speaking an electro-motive field) produced by the variation $d \boldsymbol{M}$.

We recall the expression of the vector potential $\boldsymbol{A}$ due to magnetic dipole $\boldsymbol{M} d V$ located in the origin of the cartesian axis:

$$
\vec{A}(\vec{r})=\frac{\mu_{0}}{4 \pi} \frac{(\vec{M} d V) \times \vec{r}}{r^{3}}
$$

So the part of the electric field $\boldsymbol{E}$ generated by the magnetic variation is given by:

$$
\vec{E}(\vec{r})=-\frac{\partial A(\vec{r})}{\partial t}=-\frac{\mu_{0}}{4 \pi} \frac{\left(\frac{\partial \vec{M}}{\partial t} d V\right) \times \vec{r}}{r^{3}}
$$

The time derivation is done on the magnetization $M$ only because we are in the hypothesis that all the other quantities (locations and volumes) do not vary. If we substitute (9) in (7) we obtain:

$\Delta V=\int_{L} \frac{\mu_{0}}{4 \pi} \frac{\left(\frac{\partial \vec{M}}{\partial t} d V\right) \times \vec{r}}{r^{3}} \cdot d \vec{l}=\frac{d}{d t}\left[\int_{L} \frac{\mu_{0}}{4 \pi} \frac{(\vec{M} d V) \times \vec{r}}{r^{3}} \cdot d \vec{l}\right]$

Also in this case we can take out the time derivation because all the other quantities are not time dependent.

Finally, substituting (10) in (6):

$$
d L_{M}=-\int_{L} \frac{\mu_{0}}{4 \pi} \frac{(d \vec{M} d V) \times \vec{r}}{r^{3}} \cdot d \vec{l} \cdot I_{a}
$$

If we suppose that the solenoid loops are separated each other by a quantity $\delta z_{a}$ and that $S$ is the surface of the solenoid, we can write:

$$
S=L \cdot \delta z_{a}
$$

and if the solenoid is very long with the symmetry axis coincident with the cartesian $\mathrm{z}$ axis:

$$
H_{a}=\frac{I_{a}}{\delta z_{a}}
$$

consequently:

$$
d \vec{l} \cdot I_{a}=H_{a} \cdot d S \cdot \hat{u}_{\phi}
$$

The (11) can be re-written as:

$$
d L_{M}=-\int_{S} \frac{\mu_{0}}{4 \pi} \frac{(d \vec{M} d V) \times \vec{r}}{r^{3}} \cdot \hat{u}_{\phi} \cdot H_{a} \cdot d S
$$

It is easy to find out that, because of the vector product with $\boldsymbol{r}$ and the scalar product with $\boldsymbol{u}_{\boldsymbol{\Phi}}$, the only component 
of $d M$ that does not vanish is $d M_{z}$. So the (15) can be rewritten as:

$$
d L_{M}=-\mu_{0} H_{a} d M_{z} d V \int_{S} \frac{1}{4 \pi} \frac{\hat{z} \times \vec{r}}{r^{3}} \cdot \hat{u}_{\phi} \cdot d S
$$

The integral in (16) can be easily solved considering the solenoid infinitely long:

$$
\int_{S} \frac{1}{4 \pi} \frac{\bar{z} \times \vec{r}}{r^{3}} \cdot \hat{u}_{\phi} \cdot d S^{\prime}=\int_{-\infty}^{+\infty} \frac{1}{4 \pi} \frac{2 \pi R^{2} d z}{\left(z^{2}+R^{2}\right)^{\frac{3}{2}}}=1
$$

where $R$ is the solenoid radius. We obtain the final results:

$$
d L_{M}=-\mu_{0} H_{a} d M_{z} d V
$$

In the general case, not dependent on the choice of the orientation of solenoid axis respect to the cartesian axis:

$$
d L_{M}=-\mu_{0} \vec{H}_{a} \cdot d \vec{M} \cdot d V
$$

In case of a finite volume $V$ for the considered system, the work done by the whole system, because of the magnetization variation $d M$, is then given by:

$$
d L_{M}=-\mu_{0} \int_{V} \vec{H}_{a} \cdot d \vec{M} \cdot d V
$$

The finite work $L_{M}$ due to the magnetization variation is then:

$$
L_{M}=-\mu_{0} \int_{V}^{M_{2}} \int_{M_{1}}^{H_{a}} \cdot d \vec{M} d V
$$

It is possible to note that with this approach the magnetization work $L_{M}$ is well defined also in the general case when $\boldsymbol{H}_{\boldsymbol{a}}$ is not constant on the volume $V$ : in this case $\boldsymbol{H}_{\boldsymbol{a}}$ remains inside the volumetric integral of (24). The volumetric work $l_{M}$ introduced in (1) is then demonstrated.

\section{MAGNETIZATION HEAT}

In this paragraph we calculate in a general way the heat absorbed (or given) by a system during a process involving the magnetization variation. This energy quantity, when negative, represents the heat given to the environment, and consequently, for a not reversible process, it is a term of energy dissipated by the system, for example as loss due to magnetic hysteresis or parasitic eddy currents. We will follow a classical thermodynamic approach, similar to the one done for the calculation of the magnetization work.

The heat $Q_{M}$ exchanged by the system during a magnetization process can be calculated from the first principle of thermodynamics, i.e. considering the sum of the magnetization work and the variation of the magnetic internal energy:

$$
Q_{M}=L_{M}+\Delta U_{M}
$$

where $L_{M}$ represents the work done for the magnetization, given by integration of eq. (20), and $\Delta U_{M}$ the variation of internal energy due to the magnetization contribution. In the determination of the heat $Q_{M}$ given by (25), everything is reduced to the computation of the term $\Delta U_{M}$, i.e. to the variation of internal energy due to the magnetization that goes from the initial to the final value. To evaluate $\Delta U_{M}$, some assumptions have to be made about how the energy can be "stored" in the system or in the material. Here we assume that the whole energy can be stored just for "building" and for orienting the elementary magnetic dipoles that determine the magnetization $M$ of the system. This situation particularly applies to superconductors, where the magnetization is produced by persistent currents which circulate in the material. It could also apply to other

classical problems of eddy currents, where the screening circulating currents can be substituted assuming an equivalent magnetization for the system, where $\boldsymbol{M}$ is given by (4) and $\boldsymbol{J}_{\boldsymbol{M}}$ and $\boldsymbol{J}_{\boldsymbol{S} M}$ represent the screening currents.

In this situation the internal energy stored by the system $U_{M}$ can be considered as the sum of two values: the first term represents the magnetic energy necessary to orientate the elementary dipoles (intended as $\boldsymbol{M} d V$ ) for the effect of the external magnetic field $\boldsymbol{H}_{\boldsymbol{a}}$; the second term instead represents the stored magnetic energy by itself: it could be seen as the energy necessary to "build" the magnetic system with magnetization $\boldsymbol{M}$, without the presence of any external magnetic field.

\section{A. Magnetic stored energy for orientation}

If we call $U_{M A}$ the term of energy necessary to orientate the dipoles, it can be calculated directly by elementary electromagnetic consideration: the elementary magnetic dipole $M d V$ is subjected to a torque with module $T_{\vartheta}$ given by:

$$
T_{\vartheta}=-B \cdot M d V \cdot \sin \vartheta
$$

where $\vartheta$ is the angle from $B$ direction and $\boldsymbol{M}$ direction. The work $d L$ necessary to orientate the dipole in the direction $\vartheta$ (assuming as initial position the one with the two vectors perpendicular, i.e. $\vartheta=\pi / 2$ ) is:

$$
d L=\int_{\pi / 2}^{\vartheta} T_{\vartheta} d \vartheta^{\prime}=B \cdot M d V \cdot \cos \vartheta=\vec{B} \cdot \vec{M} d V
$$

Since the energy stored to orientate the system $U_{M A}$ is equal to the work done, it follows:

$$
U_{M_{A}}=L=\int_{V} \vec{B} \cdot \vec{M} d V
$$

$\boldsymbol{B}$ is the external field, in which the system is immersed, leading:

$$
\vec{B}=\mu_{0} \vec{H}_{a}
$$

Consequently:

$$
U_{M_{A}}=\mu_{0} \int_{V} \vec{H}_{a} \cdot \vec{M} d V
$$

The differential form of (28) is then:

$$
d U_{M_{A}}=\mu_{0} \int_{V} \vec{H}_{a} d \vec{M} \cdot d V+\mu_{0} \int_{V} d \vec{H}_{a} \vec{M} \cdot d V
$$

\section{B. Magnetic energy to "build" the magnetic system}

If we call this term of internal energy $U_{M B}$, we can think to compute it from the vector potential $\boldsymbol{A}$. In the quasi-static approximation, the stored magnetic energy is given by:

$$
U_{M_{B}}=\frac{1}{2} \int_{V} \vec{A} \cdot \vec{J} \cdot d V+\frac{1}{2} \int_{S_{v}} \vec{A} \cdot \vec{J}_{s} \cdot d S
$$

where $\boldsymbol{J}$ and $\boldsymbol{J}_{\boldsymbol{s}}$ represent respectively the volumetric currents and the superficial current in the volume $V$ and on the surface $S_{v}$ of the system, and $\boldsymbol{A}$ is the vector potential due to the magnetization of the system. The volumetric and superficial currents $\boldsymbol{J}$ and $\boldsymbol{J}_{\boldsymbol{s}}$ are the ones given by the magnetization of the system, i.e. they are the fictitious or the real currents equivalent to the magnetization description, which can be calculated from eq. (4). Consequently eq. (32) can be written as:

$$
U_{M_{B}}=\frac{1}{2} \int_{V} \vec{A} \cdot \operatorname{rot} \vec{M} d V+\frac{1}{2} \int_{S_{v}} \vec{A} \cdot[\vec{M} \times \vec{n}] d S
$$

or: 


$$
U_{M_{B}}=\frac{1}{2} \int_{V} \vec{A} \cdot \operatorname{rot} \vec{M} d V+\frac{1}{2} \int_{S_{v}} \vec{n} \cdot[\vec{A} \times \vec{M}] d S
$$

Applying the divergence theorem on last term:

$$
U_{M_{B}}=\frac{1}{2} \int_{V}[\vec{A} \cdot \operatorname{rot} \vec{M} d V+\operatorname{div}(\vec{A} \times \vec{M})] d V
$$

and for a well-known vector identity:

$$
U_{M_{B}}=\frac{1}{2} \int_{V} \vec{M} \cdot \operatorname{rot} \vec{A} d V
$$

which finally gives:

$$
U_{M_{B}}=\frac{1}{2} \int_{V} \vec{M} \cdot \vec{B} d V
$$

Because we had stated that $\boldsymbol{A}$ was the vector potential due to the magnetization of material only, now we can comprehend that $\boldsymbol{B}$ in (37) is the field produced by the magnetization $\boldsymbol{M}$ in the volume $V$, i.e.. ${ }^{2}$

$$
\vec{B}=\mu_{0} \vec{H}_{m}+\mu_{0} \vec{M}
$$

where $\boldsymbol{H}_{\boldsymbol{m}}$ is the magnetic field produced by the magnetization of the volume, usually called as "demagnetizing" field. The de-magnetizing field $\boldsymbol{H}_{\boldsymbol{m}}$ is given from the "Coulombian" integration of the fictitious magnetic charges, given by (5), which are present on the volume $V$ and on the surface $S_{v}$ of the volume, and which, in analogy to electrostatics, produce $\boldsymbol{H}_{\boldsymbol{m}}$ [15]. Consequently we can write:

$$
\vec{H}_{m}(\vec{r})=-\operatorname{grad}\left\{\frac{1}{4 \pi} \int_{S_{V}} \frac{\vec{M}^{\prime} \cdot \vec{n}^{\prime}}{\left|\vec{r}-\vec{r}^{\prime}\right|} \cdot d S^{\prime}-\frac{1}{4 \pi} \int_{V} \frac{d i v \vec{M}^{\prime}}{\left|\vec{r}-\vec{r}^{\prime}\right|} \cdot d V^{\prime}\right\}
$$

For brevity here we assume that the symbol ' in any quantity has the meaning to specify explicitly that the quantity is dependent by the $\boldsymbol{r}$ ' only, i.e. for example $\boldsymbol{M}^{\boldsymbol{\prime}} \equiv \boldsymbol{M}\left(\boldsymbol{r}^{\prime}\right)$ and $\boldsymbol{M} \equiv \boldsymbol{M}(\boldsymbol{r})$. Applying the divergence theorem and a vector identity, eq. (39) becomes:

$$
\vec{H}_{m}(\vec{r})=-\operatorname{grad}\left\{\frac{1}{4 \pi} \int_{V} \frac{\vec{M}^{\prime} \cdot\left(\vec{r}-\vec{r}^{\prime}\right)}{\left|\vec{r}-\vec{r}^{3}\right|^{3}} \cdot d V^{\prime}\right\}
$$

Using (37) and (38), the variation $d U_{M B}$ becomes:

$d U_{M_{B}}=\mu_{0} \int_{V} \vec{M} \cdot d \vec{M} d V+\frac{\mu_{0}}{2}\left(\int_{V} \vec{M} \cdot d \vec{H}_{m} d V+\int_{V} \vec{H}_{m} \cdot d \vec{M} d V\right)$

the operator grad in (40) can move inside the volume integral, and it derives only the not-primate variable $\boldsymbol{r}$. The last volume integral in the parenthesis of (41) can be written now as:

$$
-\int_{V} \frac{1}{4 \pi} \int_{V} \operatorname{grad} \frac{\vec{M}^{\prime} \cdot\left(\vec{r}-\vec{r}^{\prime}\right)}{\left|\vec{r}^{\prime}-\vec{r}\right|^{3}} \cdot d \vec{M} \cdot d V^{\prime} \cdot d V
$$

and then, executing explicitly the derivatives:

$$
-\int_{V} \frac{1}{4 \pi} \int_{V}\left\{\frac{\vec{M} \cdot d \vec{M}}{\left|\vec{r}^{\prime}-\vec{r}\right|^{3}}-\frac{3\left[\vec{M}^{\prime} \cdot\left(\vec{r}-\vec{r}^{\prime}\right)\left[d \vec{M} \cdot\left(\vec{r}-\vec{r}^{\prime}\right)\right]\right.}{\left|\vec{r}^{\prime}-\vec{r}\right|^{5}}\right\} \cdot d V^{\prime} \cdot d V
$$

By reverting the order of the two volumetric integrals, the (43) can be written as:

$$
-\int_{V} \frac{1}{4 \pi} \int_{V} \operatorname{grad} \frac{d \vec{M} \cdot\left(\vec{r}^{\prime}-\vec{r}\right)}{\left|\vec{r}^{\prime}-\vec{r}\right|^{3}} \cdot \vec{M}^{\prime} \cdot d V \cdot d V^{\prime}
$$

\footnotetext{
2 The demonstration could be performed in a slight different but equivalent way: if the vector potential $\boldsymbol{A}$ in eq. (32) had included also the external field $H_{a}$, the magnetic flux $B$ in (38) would have included also the term $\mu_{0} H_{a}$. In this situation the splitting of total internal energy $U_{M}$ in two parts $U_{M A}$ and $U_{M B}$ wouldn't have been necessary.
}

which is equal to the first volume integral in the parenthesis of (41). Finally we obtain:

$$
d U_{M_{B}}=\mu_{0} \int_{V} \vec{M} \cdot d \vec{M} d V+\mu_{0} \int_{V} \vec{H}_{m} \cdot d \vec{M} d V
$$

If we go back to (25), the heat $Q_{M}$ absorbed during the magnetization process is:

$$
Q_{M}=L_{M}+\Delta U_{M_{A}}+\Delta U_{M_{B}}
$$

and in the differential form:

$$
d Q_{M}=d L_{M}+d U_{M_{A}}+d U_{M_{B}}
$$

where $d L_{M}, d U_{M^{A}}$ and $d U_{M B}$ are given respectively by (20), (31) and (46). For the linearity of the volume integral operator, it holds:

$$
\begin{aligned}
& d Q_{M}=\mu_{0} \int_{V}\left\{-\vec{H}_{a} d \vec{M}+\left(\vec{H}_{a} d \vec{M}+\vec{M} d \vec{H}_{a}\right)+\left(\vec{M} d \vec{M}+\vec{M} d \vec{H}_{m}\right)\right) d V \\
& =\mu_{0} \int_{V}\left\{\vec{M} d \vec{H}_{a}+\vec{M} d \vec{H}_{m}+\vec{M} d \vec{M}\right\} d V
\end{aligned}
$$

The constitutive relation between $\boldsymbol{B}, \boldsymbol{H}$ and $\boldsymbol{M}$ is:

$$
\vec{B}=\mu_{0}(\vec{H}+\vec{M})
$$

where:

$$
\vec{H}=\vec{H}_{a}+\vec{H}_{m}
$$

So (49) becomes:

$$
d Q_{M}=\int_{V} \vec{M} d \vec{B} d V
$$

If we rely (52) for a finite variation of heat:

$$
Q_{M}=\int_{B_{1} V}^{B_{2}} \int_{V} \vec{M} \cdot d \vec{B} d V=\int_{V}\left[\int_{B_{1}}^{B_{2}} \vec{M} \cdot d \vec{B}\right] d V
$$

According to the usual thermodynamic convention, the heat $Q_{M}$ represents an energy absorbed by the system. As a consequence, if in the magnetization process we consider that the heat is going out from the material (as in all the cases where the energy is dissipated), the heat $Q_{\text {Mext }}$ is:

$$
Q_{\text {Mext }}=-\int_{V}^{[}\left[\int_{B_{1}}^{B_{2}} \vec{M} \cdot d \vec{B}\right] d V
$$

It descends directly that the instantaneous volumetric heat-power exchanged during the process $p_{\text {Mext }}$ is then:

$$
p_{\text {Mext }}=-\vec{M} \cdot \frac{d \vec{B}}{d t}
$$

If the power density is intended as averaged over the volume $V$, eq (55) can be re-written as:

$$
p_{\text {Mext }}=-\frac{1}{V} \int_{V} \vec{M} \cdot \frac{d \vec{B}}{d t} d V
$$

Generally in superconductor hysteresis curves and in eddy currents problems the sign of magnetization $\boldsymbol{M}$ is opposite to the sign of the variation of $\boldsymbol{B}$ : this leads to a positive dissipation $p_{\text {Mext }}$.

It is useful to note that eq. (47) assures that the calculation of heat over a closed loop gives again the same results of the first equivalence of (2), because $U_{M A}$ and $U_{M B}$ are state functions, which depend on the state of the system only. Similarly, the integration of (47) over a closed loop gives exactly the second equivalence of (2) (with $H=H_{a}$, in agreement with [8]), because the last two terms of (49) are equal to $\Delta U_{M B}$ which must vanish in a closed loop. 


\section{APPLICATIONS TO HYSTERESIS LOSSES IN SUPERCONDUCTORS}

We apply the results obtained in eq. (54) to calculate the hysteresis losses in classical examples of configurations with II type superconductors.

\section{A. Slab parallel to the field}

We assume to work with the one-dimensional model of an infinitely long (z-direction) and infinitely high (ydirection) slab of superconductor, centred in the origin of the Cartesian axes, with a thickness of $2 a$ (x-direction), and an external field $H_{a}$ parallel to y direction. The analytical calculation of the volumetric losses $q$ during closed loop with a total external field variation $H_{t o t}=B_{\text {tot }} / \mu_{0}$ is well known and is based on the so called critical state model [16]; here we summarize the results reported in [5] for volumetric loss $q$ along a cycle:

$$
q=\left\{\begin{array}{cc}
\frac{B_{t o t}{ }^{2}}{2 \mu_{0}} \cdot \frac{\beta}{3} & \beta \leq 1 \\
\frac{B_{t o t}{ }^{2}}{2 \mu_{0}}\left(\frac{1}{\beta}-\frac{2}{3 \beta^{2}}\right) & \beta>1
\end{array}\right.
$$

where the ratio $\beta=B_{t o t} /\left(2 \mu_{0} J_{c} a\right)$ represents the fraction of penetration of the flux field inside the slab $(\beta \geq 1$ means full penetration), and $J_{c}$ is the critical current density of the slab, which is approximated constant during the field variation. The relation (57) is calculated with a classical method, integrating the electric power density $J_{c} E$ over the slab volume and considering a complete cycle of variation for the external field. Many other classical texts reach the same results using the second equivalence of eq. (2), or calculating total energy variation over cycle [10-12]. Again in this way the average dissipation per cycle can be computed.

To calculate the loss along the path using the new eq. (54), we need to find $M$ and $B$. Considering an external field $H_{a}$ which is swinging from the highest value $J_{c} a \beta$ to the lowest value $-J_{c} a \beta$, the magnetization $M$ in the slab is different for a partial or for fully penetrated field. According to the critical state model, for a partial penetrated field $(\beta \leq 1)$, the magnetization assumes a constant value in the central zone of the slab, whereas decreases or increases linearly (depending by the sign of the screening persistent current $J_{c}$ ) in the zone where the field penetrates. Using the normalized variable $\gamma=2 \mu_{0} H_{a} / B_{t o t}=H_{a} /\left(J_{c} a \beta\right)$, it holds:

$$
M(x)=\left\{\begin{array}{cc}
-J_{c} a \beta \gamma & 0 \leq x \leq a(1-\beta) \\
J_{c} a \beta\left[(1-\gamma)-\frac{1}{\beta}\left(1-\frac{x}{a}\right)\right] & a(1-\beta)<x \leq a \frac{2-(1-\gamma) \beta}{2} \\
J_{c} a\left(1-\frac{x}{a}\right) & a \frac{2-(1-\gamma) \beta}{2}<x \leq a
\end{array}\right.
$$

where $\gamma$ is swinging from 1 to -1 in the first half-cycle and we represent only the half space with $x \geq 0$ for the symmetry of system.

Because the de-magnetizing field $H_{m}$ is zero, $B=\mu_{0} H_{a}+\mu_{0} M$. The dependence of $M$ in eq. (58) respect to the variable $x$, is linked to the variable $\gamma$, consequently it necessary to integrate first in $x$ and later in $\gamma$. Eq. (52) becomes:

$$
d Q_{M}=\mu_{0} \int_{V} M\left(d M+d H_{a}\right)
$$

The volumetric heat exchange due to an infinitely small variation $d \gamma$ of the normalized-field is then:

$$
d q_{M}=\frac{\mu_{0}}{a} \int_{0}^{a} M\left(\frac{d M}{d \gamma}+\frac{d H_{a}}{d \gamma}\right) d x \cdot d \gamma
$$

where $\frac{d H_{a}}{d \gamma}=J_{c} a \beta$, and $\frac{d M}{d \gamma}$ have to be calculated from eq. (58). Eq. (60) becomes:

$$
d q_{M}=\frac{B_{t o t}{ }^{2}}{2 \mu_{0}} \frac{\beta}{16}(1-\gamma)^{2} d \gamma
$$

and finally, integrating eq. (61) from 1 to $\gamma$ and changing the sign, to find the volumetric heat going out from the system:

$$
q_{\text {Mext }}=\frac{B_{t o t}{ }^{2}}{2 \mu_{0}} \frac{\beta}{48}(1-\gamma)^{3}
$$

The (63) represents the dissipated heat during the external field variation from the initial field $J_{c} a \beta$ (i.e. $\gamma=1$ ) to $H_{a} \geq-J_{c} a \beta$; when $H_{a}=-J_{c} a \beta$ (i.e. $\gamma=-1$ ) the system has accomplished half cycle, and the accumulated loss is exactly half value of the one in (57) with $\beta \leq 1$.

The instantaneous power loss $p_{\text {Mext }}$ can be calculated by deriving (63) respect to the time (only $\gamma$ depends on the time), which, returning from the a-dimensional variable $\gamma$ to $H_{a}$, can be re-written as:

$$
p_{\text {Mext }}=-\frac{\mu_{0}}{8 J_{c} a}\left(\frac{B_{t o t}}{2 \mu_{0}}-H_{a}\right)^{2} \dot{H}_{a}
$$

The case with $\beta>1$ gives different values for $M(x)$; in fact the magnetization has not a flat plateau in the slab and it varies until $H_{a}$ has decreased of $2 J_{c} a$ from the initial maximum value (i.e. $1-2 / \beta \leq \gamma \leq 1$ ); later it remains constant until the half cycle is completed: So for $1-2 / \beta \leq \gamma \leq 1$ :

$$
M(x)=\left\{\begin{array}{cc}
J_{c} a \beta \gamma\left[1-\gamma+\frac{1}{\beta}\left(\frac{x}{a}-1\right)\right] & 0 \leq x \leq a \frac{2-(1-\gamma) \beta}{2} \\
J_{c} a\left(1-\frac{x}{a}\right) & a \frac{2-(1-\gamma) \beta}{2}<x \leq a
\end{array}\right.
$$

Whereas for the last part of field variation, i.e. when $-1 \leq \gamma<1-2 / \beta$ :

$$
M(x)=J_{c} a\left(1-\frac{x}{a}\right)
$$

Eq. (65) has to be used in eq. (60) to find the accumulated heat in the first part of field variation $(\gamma$ decreasing from 1 to $1-2 / \beta$ ), whereas eq. (66) has to be used in eq. (60) to find the accumulated heat when $\gamma$ decreases from $1-2 / \beta$ to -1 . After the integration first in $x$ and later in $\gamma$, we obtain:

$q_{\text {Mext }}=\left\{\begin{array}{cc}\frac{B_{t o t}{ }^{2}}{2 \mu_{0}} \frac{\beta}{48}(1-\gamma)^{3} & 1-\frac{2}{\beta} \leq \gamma \leq 1 \\ \frac{B_{t o t}{ }^{2}}{2 \mu_{0}}\left(\frac{1-\gamma}{4 \beta}-\frac{1}{3 \beta^{2}}\right) & -1 \leq \gamma \leq 1-\frac{2}{\beta}\end{array}\right.$ 
Again eq. (67) represents the dissipated heat by the system when the normalized-field $\gamma$ decreases from 1 to the generic $\gamma \geq-1$; when $\gamma=-1$ the system has accomplished half cycle and the accumulated loss is exactly half value of the one in (57) with $\beta>1$.

The instantaneous power loss $p_{\text {Mext }}$ in the explicitly form is then:

$$
p_{\text {Mext }}=\left\{\begin{array}{cc}
-\frac{\mu_{0}}{8 J_{c} a}\left(\frac{B_{\text {tot }}}{2 \mu_{0}}-H_{a}\right)^{2} \dot{H}_{a} & J_{c} a(\beta-2) \leq H_{a} \leq J_{c} a \beta \\
-\frac{\mu_{0} J_{c} a}{2} \dot{H}_{a} & -J_{c} a \beta \leq H_{a} \leq J_{c} a(\beta-2)
\end{array}\right.
$$

This dissipations could be alternately calculated classically integrating the electric power density $J_{c} E$ over the slab volume and considering a partial variation of the external field, instead of a complete cycle of variation, obtaining the same analytical functions of eq. (63), (64), (67) and (68). An example can be found in [6], whose results of the power losses are in perfect agreement with (64) and (68).

The Fig. 1 represents the behavior of the accumulated loss $q_{\text {Mext }}$ (normalized respect to the factor $B_{t o t}{ }^{2} / 2 \mu_{0}$ ) given by (63) and (67) for different values of the penetration factor $\beta$.

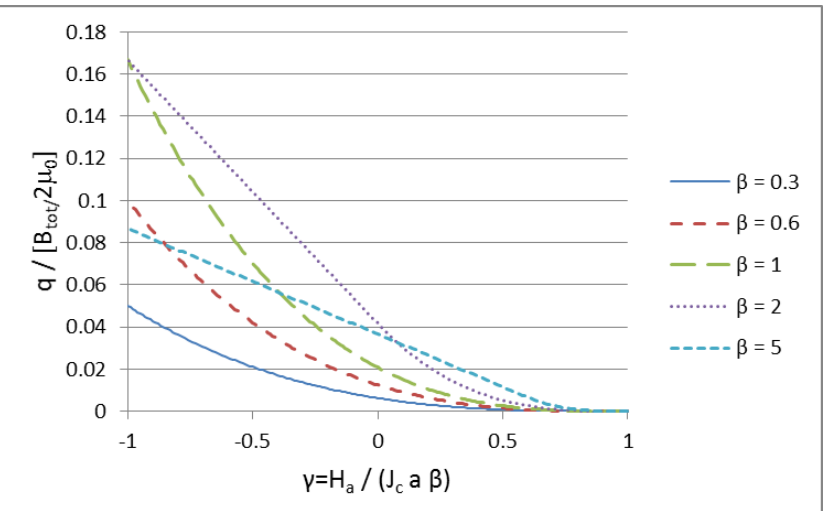

Fig. 1. Normalized accumulated heat starting from $\gamma=1$ up to $\gamma=-1$ (half cycle), for different values of the penetration factor $\beta$. The reading of the graph has to be done considering the $\mathrm{x}$-axis moving from the maximum value $(\gamma=1)$ up to lower values.

Observing the slope of the curves in Fig. 1 or using eq. (64) and (68), it is easy to note that for a linear ramp of $H_{a}$, the power is lower at the beginning of the ramp and increases with the square of the field variation, until, for $\beta>1$, it remains constant when $\Delta H_{a}<-2 J_{c} a$. Because in most practical cases the condition $\left|\Delta H_{a}\right|>2 J_{c} a$ is reached at the very beginning of the field variation, the power loss can be usually considered constant during all the cycle.

\section{B. Cylinder parallel to the field}

In this example we give the example of the onedimensional model of an infinitely long (z-direction) cylinder of superconductor, centered in the origin of the axes, with radius $a$ and an external field $H_{a}$ parallel to zdirection. Again, the analytical calculation of the volumetric losses $q$ during a closed loop with a total external field variation $H_{t o t}=B_{t o t} / \mu_{0}$, based on the so called critical state model can be found for example in [5], and here we just summarize the results:

$$
q= \begin{cases}\frac{B_{t o t}{ }^{2}}{2 \mu_{0}}\left\{\frac{2 \beta}{3}-\frac{\beta^{2}}{3}\right\} & \beta \leq 1 \\ \frac{B_{t o t}{ }^{2}}{2 \mu_{0}}\left(\frac{2}{3 \beta}-\frac{1}{3 \beta^{2}}\right) & \beta>1\end{cases}
$$

where again the ratio $\beta=B_{\text {tot }} /\left(2 \mu_{0} J_{c} a\right)$ represents the fraction of penetration of the flux field inside the cylinder ( $\beta \geq 1$ means full penetration), and $J_{c}$ is the critical current density of the cylinder, which is approximated constant during the field variation. The relation (69) is obtained with classical methods, i.e. integrating the electric power density $J_{c} E$ over the cylinder volume and considering a complete cycle of variation for the external field.

To calculate the dissipated heat using eq. (54), we need to find $M$ and $B$. Considering an external field $H_{a}$ which is swinging from the highest value $J_{c} a \beta$ to the lowest value $-J_{c} a \beta$, the magnetization $M$ in the cylinder is different for a partial or for fully penetrated field. According to the critical state model, for a partial penetrated field $(\beta \leq 1)$, the magnetization assumes a constant value in the central zone of the cylinder, whereas decreases or increases linearly (depending by the sign of the screening persistent current $J_{c}$ ) in the zone where the field penetrates. Using the normalized variable $\gamma=2 \mu_{0} H_{a} / B_{\text {tot }}=H_{a} /\left(J_{c} a \beta\right)$, it holds:

$$
M(r)=\left\{\begin{array}{cc}
-J_{c} a \beta \gamma & 0 \leq r \leq a(1-\beta) \\
J_{c} a \beta\left[(1-\gamma)-\frac{1}{\beta}\left(1-\frac{r}{a}\right)\right] & a(1-\beta)<r \leq a \frac{2-(1-\gamma) \beta}{2} \\
J_{c} a\left(1-\frac{r}{a}\right) & a \frac{2-(1-\gamma) \beta}{2}<r \leq a
\end{array}\right.
$$

where $\gamma$ is swinging from 1 to -1 in the first half-cycle.

Because the de-magnetizing field $H_{m}$ is zero, $B=\mu_{0} H_{a}+\mu_{0} M$. The dependence of $M$ in eq. (70) respect to the variable $r$, is linked to the variable $\gamma$, consequently it is necessary to integrate first in $r$ and later in $\gamma$. From eq. (54) we can write:

$$
d q_{M}=\frac{\mu_{0}}{\pi a^{2}} \int_{0}^{a} M\left(\frac{d M}{d \gamma}+\frac{d H_{a}}{d \gamma}\right) 2 \pi r d r \cdot d \gamma
$$

where $\frac{d H_{a}}{d \gamma}=J_{c} a \beta$, and $\frac{d M}{d \gamma}$ have to be calculated from eq. (70). Eq. (71) becomes:

$$
d q_{M}=\frac{B_{t o t}{ }^{2}}{2 \mu_{0}}\left\{\frac{\beta}{8}(1-\gamma)^{2}-\frac{\beta^{2}}{24}(1-\gamma)^{3}\right\} d \gamma
$$

Integrating eq. (72) from 1 to $\gamma$ and changing the sign, we find the volumetric heat going out from the cylinder:

$$
q_{\text {Mext }}=\frac{B_{\text {tot }}{ }^{2}}{2 \mu_{0}}\left\{\frac{\beta}{24}(1-\gamma)^{3}-\frac{\beta^{2}}{96}(1-\gamma)^{4}\right\}
$$

The (73) represents the dissipated heat during the external field variation from the initial field $J_{c} a \beta$ (i.e. $\gamma=1$ ) to $H_{a} \geq-J_{c} a \beta$; when $H_{a}=-J_{c} a \beta$ (i.e. $\gamma=-1$ ) the system has accomplished half cycle and this accumulated loss is exactly half value of the one in (69) with $\beta \leq 1$.

The case with $\beta>1$ gives the following values for $M(r)$ : for $1-2 / \beta \leq \gamma \leq 1$ : 


$$
M(r)=\left\{\begin{array}{cc}
J_{c} a \beta \gamma\left[1-\gamma+\frac{1}{\beta}\left(\frac{r}{a}-1\right)\right] & 0 \leq r \leq a \frac{2-(1-\gamma) \beta}{2} \\
J_{c} a\left(1-\frac{r}{a}\right) & a \frac{2-(1-\gamma) \beta}{2}<r \leq a
\end{array}\right.
$$

Whereas for the last part of field variation, i.e. when $-1 \leq \gamma<1-2 / \beta$ :

$$
M(r)=J_{c} a\left(1-\frac{r}{a}\right)
$$

Eq. (74) has to be used in eq. (71) to find the accumulated heat in the first part of field variation $(\gamma$ decreasing from 1 to $1-2 / \beta$ ), whereas eq. (75) has to be used in eq. (71) to find the accumulated heat when $\gamma$ decreases from $1-2 / \beta$ to -1 . After the integration first in $r$ and later in $\gamma$, we obtain:

$$
q_{\text {Mext }}=\left\{\begin{array}{cc}
\frac{B_{\text {tot }}{ }^{2}}{2 \mu_{0}}\left\{\frac{\beta}{24}(1-\gamma)^{3}-\frac{\beta^{2}}{96}(1-\gamma)^{4}\right\} & 1-\frac{2}{\beta} \leq \gamma \leq 1 \\
\frac{B_{t o t}{ }^{2}}{2 \mu_{0}}\left(\frac{1-\gamma}{6 \beta}-\frac{1}{6 \beta^{2}}\right) & -1 \leq \gamma \leq 1-\frac{2}{\beta}
\end{array}\right.
$$

Again this represents the heat dissipated by the system when the normalized-field $\gamma$ decreases from 1 to the generic $\gamma \geq-1$; when $\gamma=-1$ the system has accomplished half cycle and the accumulated loss is exactly half value of the one in (69) for the case $\beta>1$.

This dissipation could be alternately calculated classically integrating the electric power density $J_{c} E$ over the cylinder volume and considering a partial variation of the external field, instead of a complete cycle of variation, obtaining the same analytical functions of eq. (73) and eq.(76).

\section{APPLICATIONS TO INTER-FILAMENTS LOSSES}

In this paragraph we consider as example of application of eq. (54) and (55) the calculation of losses due to interfilament coupling currents in composite superconductors induced by a transverse external field. A classic method to calculate this loss for uniform field variation is considering the Joule effect (ohmic dissipation) [17]. Here we recall the final results following the notation reported in [5]. The integration of the joule power over the strand gives the volumetric dissipated power $p$ for inter filaments currents:

$$
p=\frac{2 \dot{B}_{i}^{2}}{\mu_{0}} \tau
$$

where the time constant $\tau$ is related to the filament twist pitch $L$ and to the effective transverse resistivity of the matrix $\rho_{e t}$ by means of:

$$
\tau=\frac{\mu_{0}}{2 \rho_{e t}}\left(\frac{L}{2 \pi}\right)^{2}
$$

In eq. (77) $B_{i}$ is the total internal field inside the strands, due to external field variation and to the a.c. currents, and in the above model it is assumed constant. The screening inter-filament currents produce an equivalent average magnetization for the strands, which according to the same model in [5] is:

$$
M=-\frac{2 \dot{B}_{i}}{\mu_{0}} \tau
$$

For calculating the same power using (55) we can use (79), were $B=B_{i}$. It is then trivial to find the same result of (77).

\section{FINAL REMARKS}

This alternative way to calculate heat or power loss in systems which can be treated as magnetized volume may be of great practical interest, for the simplicity of the relations (54) and (55). In the scientist community of superconducting magnet design these relations are used since few years [18]: however a general and theoretical assessment of their validity in any situation was lacking, especially when intended as instantaneous heat or instantaneous power and not only as average power over a cycle. The advantage respect to other classic methods is that if the curve $\boldsymbol{M}$ vs. $\boldsymbol{B}$ of the system is known, the calculation is quite direct. Of course this method is consistent with all other classic methods (the integration of $\boldsymbol{J} \cdot \boldsymbol{E}$, for example) because they all descends from the same Maxwell equations and from the same material properties (critical state model for II type superconductor, for example).

If we consider the dependence of $\boldsymbol{B}$ respect to the current $I$, a useful derivation of eq (55) to calculate the total dissipated power is:

$$
P_{\text {Mext }}=-\frac{d I}{d t} \int_{V} \vec{M} \cdot \frac{d \vec{B}}{d I} d V
$$

If a linear relation between $\boldsymbol{B}$ in volume $V$ and the magnetic field $B_{0}$ in a specific point (as the bore of the magnet, for example) can be assumed (this is a very common case and it is a valid approximation in the region $V$ occupied by superconducting coils, where the effect of the non-linearity of $\boldsymbol{B}$ for the magnetization contribution is much marginal), it holds:

$$
\frac{d \vec{B}}{d I}=\frac{\vec{B}}{B_{0}} \cdot \frac{d B_{0}}{d t}
$$

So (80) gives:

$$
P_{\text {Mext }}=-\frac{d I}{d t} \cdot \frac{d B_{0}}{d I} \cdot \frac{1}{B_{0}} \int_{V} \vec{M} \cdot \vec{B} d V
$$

where $d B_{0} / d I$ represents the slope of the load line in the point where $B_{0}$ is evaluated. The computation of the losses becomes now direct: when the dependence of $M$ vs. $B$ is given, the calculation can be easily done, for example with usual codes for magnetostatic analysis, integrating $M(B) \cdot B$ ( $\boldsymbol{M}$ and $\boldsymbol{B}$ are usually parallel) over the volume $V$. An example of application of this method is given in [19].

\section{CONCLUSIONS}

With a classical approach we have demonstrated how to calculate heat exchange during magnetizing process, for those systems where the internal energy due the magnetization can be stored in the elementary magnetic dipole orientation and variation only. It applies particularly for systems where the magnetization is due to screening currents like in superconductors or in eddy current problem. With the two examples in sections IV and V we have shown the agreement of the results with other classical approaches. In section VI we have shown the practical interest of this approach for the community 
devoted to magnet design. The present method to calculate heat in magnetic system is general and can be applied independently by type of field variation, as periodic or notperiodic variation, "fast" or "slow" variation. In principle it applies both for bulk and for composite materials, as well for twisted superconducting strands and cables, provided that the magnetization $\boldsymbol{M}$ and the flux field $\boldsymbol{B}$ can be calculated. It applies particularly well for all transient phenomena and for all the cases in which the magnetic variation is not periodic, so that the losses cannot be calculated as it is usually done in periodic a.c. problems. Despite their simplicity, relations (54) and (55) may offer an alternative and elegant method for calculating heat exchange or heat dissipation in magnetized materials.

\section{REFERENCES}

[1] Mark W. Zemansky, Heat and Thermodynamics ( 5th.ed.) McGrawHill Book Company Inc. 1968, p.62

[2] Georg Busch, Horst Schade, Lectures on Solid State Physics, International Series in Natural Philosophy Vol. 79 Pergamon Press 1976 p.409

[3] A.B. Pippard, Elements of classical thermodynamics for advanced students of physics, Cambridge at the university press 1966, p.24

[4] Herbert B. Callen, Thermodynamics and introduction to thermostatics (2nd ed.) John Wiley \& sons Inc. 1985, p.479

[5] M.N. Wilson, Superconducting Magnets, Clarendon Press Oxford, 1983, pp.161-165,176

[6] W.J. Carr, Jr., AC Loss and Macroscopic Theory of Superconductors: second edition, CRC Press, 2001, p.12,51,67

[7] M. Ashkin, Flux distribution and hysteresis loss in a round superconducting wire for the complete range of flux penetration, Journal of Applied Physics Vol. 50, No. 11, 1979, p. 7063
[8] Francesco Grilli, Enric Pardo, Antti Stenvall, Doan N. Nguyen, Weijia Yuan and Fedor Gömöry, " Computation of Losses in HTS Under the Action of Varying Magnetic Fields and Currents", IEEE Trans. Appl. Supercond. vol 24, no. 1, 2014.

[9] V.B Zenkevitch, A.S. Romanyuk and Zheltov Losses in composite superconductors at high levels of magnetic field excitation: part 1, Cryogenics, Vol. 20 No. 12, 1980, p.705

[10] H. Brechna, Superconducting magnet system, Vol. 18 of Technische Physik in Einzeldarstellungen, Springer, 1973, p. $241 \mathrm{ff}$.

[11] A.M. Campbell and J.E. Evetts, Critical currents in superconductors, Taylor and Francis, 1972, p. 74

[12] J.E.C. Williams, Superconductivity and its applications, Pion Limited, 1970, p. $138 \mathrm{ff}$

[13] Y. Kato, M. Hanawaka and K. Yamafuji, Flux Penetration into a Wire of a Nonideal Type II Superconductor in a Transverse Magnetic Field, Japanese Journal of Applied Physics, Vol. 15, No. 4, 1976, p.699

[14] W.J. Carr Jr, Hysteresis loss in a coated conductor subject to combined applied magnetic field and transport current, Supercond. Sci. Technol. 19, 2006, p.457

[15] E. Durand, Magnétostatique, Masson Paris, 1968, p. 20 ff.

[16] Bean, Magnetization of Hard Superconductors, Phys. Rev. Lett. 8, $250,1962$.

[17] G.H. Morgan, Theoretical behavior of twisted multicore superconducting wire in a time-varying uniform magnetic field, Journal of Applied Physics, Vol.41, No.9, 1970, p.3674

[18] M.N.Wilson, Lectures on Magnetization and ac losses for superconducting magnets, slides on the web, for example: http://indico.cern.ch/event/356897/contribution/62/attachments/7100 05/974655/JUAS 15 lect 2 magn cables ac.pdf , 2015, slide 24 25

[19] M. Sorbi, F. Alessandria, G. Bellomo, S. Farinon, U. Gambardella, P. Fabbricatore, and G. Volpini, Field Quality and Losses for the 4.5 $\mathrm{T}$ Superconducting Pulsed Dipole of SIS300, IEEE Trans. on Applied Superconductivity, Vol. 18 N.2 June 2008, p.138-141 